${ }^{\circ}$ Entomologica Fennica. 9.XII.1993

\title{
Index to genera and species, Volume 4 (1993)
}

Acari

Amblydromella suecica (Sellnick) 96, 102

- viktorovi (Wainstein) 96, 102

Amblydromella (Seiulus) bakeri (Garman) 96, 103

- rhenana (Oudemans) 102

Amblydromella (Typhlodromus) richteri (Karg) 96, 103

Amblyseius 100, 105

- barkeri (Hughes) 105, 107

- coprophilus Karg 106

- cucumeris (Oudemans) 105, 106

- mckenziei Schuster \& Pritchard 107

- reductus Wainstein 96, 97, 105, 106

- subsolidus (Beglyarov) 97, 105

- tardi Lombardini, Kennett \& Caltagirone 104

- tenuis (Hirschmann) 105, 106

- zwoelferi (Dosse) 105, 106

Anthoseius 100, 102

- bakeri (Garman) 97, 103

- rhenanus (Oudemans) 96, 97, 102

- richteri (Karg) 97, 103

- suecicus (Sellnick) 97, 102

- viktorovi Wainstein 97, 102

Bawus talbii (Athias-Henriot) 96, 101

Dubininellus juvenis (Wainstein \& Arutunjan) 100

- macropilis (Banks) 100

Euseius 100, 105

- finlandicus (Oudemans) 96, 97, 105

Euseius (Seiulus) finlandicus (Oudemans) 95

Lauroppia ordunensis Iturrondobeitia \& Saloña 199

Medioppia 195

- beskidyensis Niemi \& Skubała sp. n. 195, 197-200

- centrodentata Gordeeva \& Niemi 195

- loksai (Schalk) 198

- minimedia Arillo \& Subías 195

- ordunensis (Iturrondobeitia \& Saloña) 195

- templadoi Pérez-Iñigo 195

- truncata Iturrondobeitia \& Saloña 195

- vera (Michelčič) 198

Moriziella escotata Subías \& Rodríguez 199

Morizoppia 195

- escotata (Subías \& Rodríguez) 195, 196

- longilamellata Subías \& Rodríguez 195, 196

- minuta Gordeeva \& Grishina 195, 196

- oreia Colloff \& Seyd 195

- sharipovi Niemi \& Skubała sp. n. 195-197

Neoseiulus barkeri Hughes 107

- subsolidus (Beglyarov) 105

- suecicus Sellnick 102

Neoseiulus (Amblyseius) canadensis (Chant \& Hansell) 105

- reductus (Wainstein) 105, 106

Neoseiulus (Typhlodromus) cucumeris (Oudemans) 106

- thripsi (MacGill) 106

- zeitunicus Wainstein \& Arutunjan 106

- zwölferi (Dosse) 106

Oppia vera Mihelčič 199
Oppiella acutirostris Pérez-Iñigo 199

Panonychus ulmi (Koch) 95

Paraseiulus 100, 101

- incognitus Wainstein \& Arutunjan 101

- ostiolatus Athias-Henriot 101

- soleiger (Ribaga) 95-97, 101

- subsoleiger Wainstein 101

- talbii (Athias-Henriot) 97, 101

- triporus (Chant \& Yoshida-Shaul) 96, 97, 101

Phytoseiulus 100, 104

Phytoseius 100

- juvenis Weinstein \& Arutunjan 100

- macropilis (Banks) 95-97, 100

- persimilis Athias-Henriot 104

- riegeli Dosse 104

Phytoseius (Seiulus) spoofi (Oudemans) 100

Proprioseiopsis 100, 104

- okanagensis (Chant) 104

Seiulus Berlese 100

- aceri (Collyer) 96, 100

- amaliae Ragusa \& Swirski 101

- bakeri Garman 103

- finlandicus Oudemans 105

- rhenanus Oudemans 102

- soleiger Ribaga 101

- spoofi (Banks) 95

Sejus macropilis Banks 100

Typhloctonus aceri (Collyer) 100

Typhlodromips (Typhlodromus) tenuis (Hirschmann) 106

Typhlodromus Scheuten 100

- aceri Collyer 100

- andrei Karg 103, 104

- bakeri (Garman) 103

- cucumeris Oudemans 106

- foenilis Oudemans 102

- gilvus Wainstein 102

- kazachstanicus Wainstein 102

- laurae Arutunjan 103

- macropilis (Banks) 100

- okanagensis levis Wainstein 104

- pritchardi Arutunjan 104

- pyri Scheuten 103

- richteri Karg 103

- soleiger (Ribaga) 101

- subsolidus Beglyarov 105

- suecicus (Sellnick) 102

- talbii Athias-Henriot 101

- tenuis Hirschmann 106

- tetramedius Zaher \& Shehata 101

- trimediosetus Xin, Liang \& Ke 101

- triporus Chant \& Yoshida-Shaul 101

- zwölferi Dosse 106

Typhlodromus (Amblyseius) finlandicus (Oudemans) 105

- okanagensis Chant 104

- okanagensis levis Wainstein 104

Typhlodromus (Neoseiulus) rhenanus (Oudemans) 102 


\section{Coleoptera}

Further 167 species of Ethiopian Dytiscidae and their synonymic names are to be found on pp. 57-94, 22 Zambian Cleridae on page 223, and 52 Carabidae as well as 186 Staphylinidae from Finnish Lapland on pp. 225-231.

Agabus paludosus (Fabricius) 14

Altica 115-119, 124, 125, 129

- aenescens Weise 118-120, 122, 124-127, 129

- carduorum (Guérin-Ménéville) 115, 117, 119, 121$125,127,129$

- carduorum cirsii Israelson 122

- carinthiaca Weise 116, 120, 123-125, 127, 129

- chamaenerii Håkan Lindberg 118, 120-127, 129

- cirsii Israelson 115, 122, 124

- engstromi J. Sahlberg 117, 119, 120, 122, 124, 125, 127,129

- longicollis (Allard) 117, 120-127, 129

- lythri Aubé 117-121, 124-127, 129

- oleracea (Linnaeus) 116-118, 121-125, 127, 129

- opacifrons Harald Lindberg 118, 121-125, 127, 129

- palustris Weise 117, 121, 123-127, 129

- quercetorum quercetorum Foudras 116, 124, 125

- quercetorum saliceti Weise 115, 117, 118, 120, 121, 124-127, 129

- tamaricis Schrank 116, 117, 121-125, 127, 129

Anacaena limbata (Fabricius) 13

Anchomenus dorsalis (Pontoppidan) 9

Anisotoma humeralis (Fabricius) 23

Apion meliloti Kirby 13

Astenus longelytratus Palm 14

Atheta 17

Atheta (Dimetrota) marcida Erichson 17

Attagenus 9

- fasciatus (Thunberg) 9

- woodroffei Halstead \& Green 9

Carabus glabratus Paykull 27-30

- violaceus Linnaeus 30

Carpathis striatus (Olivier) 23

Cercyon sternalis (Sharp) 14

Corticaria crenulata (Gyllenhal) 17

Cynaeus opacus Champion 10

Demetrias imperialis (Germar) 17

Dendrophagus crenatus Paykull 22

Dircaea quadriguttata (Paykull) 23

Dorcatoma robusta Strand 23

- substriata Hummel 23

Dromius longiceps Dejean 17

Epuraea 23, 25, 26, 133-135

- angustula (Sturm) 134

- bickhardti St.-Claire Deville 133-135

- biguttata (Thunberg) 134

- boreella (Zetterstedt) 133-135

- concurrens Sjöberg 25, 26

- convergens Reitter 25

- neglecta (Heer) 25, 26

- pygmaea (Gyllenhal) 134

- unicolor (Olivier) 134

Graphoderus cinereus (Linnaeus) 14

Gyrinus suffriani Scriba 14

Helophorus grandis Illiger 14

- griseus Herbst 14
Hydrochus megaphallus van Berge 14

Hylobius abietis (Linnaeus) 141

Liophloeus lentus Germar 15

Lordithon lunulatus (Linnaeus) 23

Microdota wireni Brundin 225, 226

Monochamus 137

- alternatus Hope 140

- carolinensis (Olivier) 140

- galloprovincialis Olivier 137-140

- sartor (Fabricius) 141

- scutellatus (Say) 140

- titillator (Fabricius) 140

Mycetophagus quadripustulatus (Linnaeus) 23

Nanosella 17

Nargus velox (Spence) 15

Ochthebius czwalinai Kuwert 14

- stockmanni Balfour-Browne 14

Oedemera croceicollis (Gyllenhal) 15

Peltis grossa (Linnaeus) 23

Philonthus pseudovarians Strand 14

Phloeopora 16

- opaca Bernhauer 16

- teres (Gravenhorst) 16

Phytobaenus amabilis F. Sahlberg 23

Tachinus basalis Erichson 16

Tetropium 141

Tomoxia bucephala Costa 23

Trachyphloeus laticollis auct. nec Boheman 18

- rectus Thomson 18

Triplax ruficeps (Fabricius) 23

- russica (Linnaeus) 23

Trogoderma angustum (Solier) 14

Typhaea 10

- desipiens Lohse 10

- stercorea (Linnaeus) 10

\section{Crustacea}

Asellus aquaticus Linnaeus 215

Polyartemia forcipata Fischer 217

\section{Diptera}

Further 81 taxa are listed on pp. 154-155, and many of them discussed on pp. 151-160.

Atherix ibis (Fabricius) 161-163

Blaesoxipha 146

Brachicoma 147, 148

- borealis Ringdahl 143, 147, 148

- devia (Fallén) 147, 148

Cheilosia 207-212

- albohirta Hellén 207-209

- brunnipennis Becker 207,210, 211

- fraterna Meigen 207

- melanura Becker 212

- parafasciata Barkalov 207, 211, 212

- reniformis Hellén 207, 209, 210

- rotundicornis Hellén 207, 210

- sapporense Shiraki 208

- sareptana Becker 210

- sichotana Stackelberg 210

- subarctica Hellén 207, 208

- velutina Loew 207, 208 
- vernalis Fallén 207, 209, 210

Echinomyia marklini Zetterstedt 147

Parasarcophaga 148

- pleskei Rohdendorf 148

Ravinia pernix (Harris) 146

Musca carnaria Linnaeus 144

- major larvarum De Geer 144

- minor larvarum De Geer 144, 146

- vivipara major De Geer 143-146

- vivipara minor De Geer 143-146

Pollenia vagabunda (Meigen) 146

Ravinia pernix (Harris) 146

- striata (Fabricius) 146

Sarcophaga 144-146

- carnaria (Linnaeus) 144, 146

- cruentata Meigen 147

- lasiostyla Macquart 146

- 'nova' Siebke 146, 147

- pleskei Rohdendorf 143, 149

- schulzi Müller 144

- similis Meade 145

- striata Siebke 146, 147

- subvicina Rohdendorf 146

- tuberosa Pandellé 148, 149

- tuberosa verticina Ringdahl 148, 149

- variegata (Scopoli) 146

- verticina Ringdahl 143, 149

Sarcophaga (Liosarcophaga) pleskei (Rohdendorf) 148

- portschinskyi (Rohdendorf) 149

Sarcophaga (Helicophagella) melanura Meigen 146

\section{Ephemeroptera}

Baetis fuscatus (Linnacus) 217

- macani Kimmins 217

- subalpinus Bengtsson 215, 217

Ephemerella ignita (Poda) 215

Heptagenia borealis Eaton 215

- joernensis Bengtsson 215, 217

- orbiticola Kluge 217

Leptophlebia marginata (Linnaeus) 215

- vespertina (Linnaeus) 215

Metretopus 213-222

- alter Bengtsson 213-221

- borealis (Eaton) 213-221

- norvegicus Eaton 215, 219

Paraleptophlebia cincta (Retzius) 217

- strandii (Eaton) 215, 217

Parameletus 220

Procloeon bifidum (Bengtsson) 215

Siphloplecton 213

Siphlonurus 214

- alternatus (Say) 215

- lacustris Eaton 215, 217

\section{Heteroptera}

In addition, 76 taxa of Miridae (subfamily Phylinae) from Iraq are listed on pp. 253-271 (however, new synonymies and new combinations on these are referred to page 253 in the index below).

Acrorrhinium 169

- conspersus Noualhier 169, 170
- elegans Linnavuori \& Al-Safadi sp. n. 171, 173

- minutissimus Linnavuori \& Al-Safadi sp. n. 172, 173, 175

Acrosternum 235-239

- apicale Linnavuori 235, 237, 238

- arabicum Wagner 235, 236, 238

- breviceps (Jakovlev) 50, 235, 236

- curticeps Linnavuori \& Al-Safadi sp. n. 235-239

- heegeri Fieber 238

- miliaris (Klug) 235-238

- millierei (Mulsant \& Rey) 50, 51, 235, 237, 238

Aelia 43

- acuminata (Linnaeus) 43

- albovittatus Fieber 43

- glebana Ferrari 43

- klugi Hahn 19

- satunini Kiritshenko 43, 44

- syriaca Horváth 43

- turanica Horváth 43, 44

Aeliomorpha sheanensis Linnavuori 44

Aeolocoris 188

- alboconspersus Reuter 188, 190

- decarinatus Linnavuori 190

- pumilus Linnavuori 190

- rufipennis Linnavuori \& Al-Safadi sp. n. 179, 187-189

Aethus hispidulus (Klug) 37, 38

- hispidulus Signoret 38

- pilosulus (Klug) 38

- rugosus (Jakovlev) 38

- sahlbergi (Reuter) 37

- setosus Walker 38

Agalliastes punctatus Fieber 233

Agatharchus tritaenia Horváth 45-47

Agramma tropidopterum Flor 19

Agraphopus femoralis Horváth 55

Agraphopus (Agraphopus) lethierryi Stål 55

- suturalis Reuter 55

Agraphopus (Leptocaraea) viridis (Jakovlev) 55

Alphocoris halophilus Linnavuori 39

Amaurocoris curtus (Brullé) 38

- orbicularis Jakovlev 38

Anchesmus rubriplaga (Walker) 51

Ancyrosoma leucogrammes (Gmelin) 41

Andrallus spinidens (Fabricius) 51

Antheminia lunulata (Goeze) 47

- pusio (Kolenati) 47

Anthocoris limbatus Fieber 19

Apodiphus amygdali (Germar) 41

Aradus 23

- crenaticollis R.F.Sahlberg 19

- laeviusculus Reuter 20

- pictus Bärensprung 20

Arenocoris intermedius (Jakovlev) 53

- waltli (Herrich-Schäffer) 53

Atomophora 190

- basipunctata Wagner 190

- sabaea Linnavuori \& Al-Safadi sp. n. 179, 189, 190

- subpallida Wagner 190

Atomoscelis signaticornis Reuter 253

Atractotomus morio J.Sahlberg 20

Badezorus 253

- signaticornis (Reuter) 253

- tomentosus (Reuter) 253

- tyrianus 253 
Bagrada abeillei Puton 48

- amoenula (Walker) 48

- cicur Horváth 48

- deserticola Horváth 48

- hilaris (Burmeister) 48

- lepida Horváth 48

- monticola Horváth 48,49

- pallens Kiritshenko 48

- persica Horváth 48

- royeri Horváth 48

- singularis Horváth 48

Bibundiella 188

Brachycarenus tigrinus (Schilling) 54

Brachynema cinctum (Fabricius) 47

- germari (Kolenati) 47

Byrsinocoris nigroscutellatus Montandon 38

Camptopus illustris Horváth 54

- lateralis (Germar) 53

Camptotylidea 189

Campylomma 241-245

- acaciae Linnavuori 241, 243

- angustior Poppius 243

- fallaciosa Linnavuori \& A1-Safadi sp. n. 241-243

- incerta Villiers 253

- longicornis Odhiambo 243

- minima Wagner 253

- oreophila Linnavuori \& Al-Safadi sp. n. 241-243

- rivulorum Linnavuori \& A1-Safadi sp. n. 241, 243, 244

- verticata Wagner 253

- viridissima Linnavuori \& Al-Safadi sp. n. 241, 244, 245

Canthophorus melanopterus (Herrich-Schäffer) 37

Capsus cuneatus Stål 233

- wagneri Remane 20

Carpocoris coreanus Distant 45

- mediterraneus mediterraneus Tamanini 46

- pudicus (Poda) 46

Centrocoris spiniger (Fabricius) 52

- volxemi (Puton) 53

Ceraleptus obtusus (Brullé) 53

Cercinthus lehmanni (Kolenati) 53

Chamaepsallus 253

- signaticornis (Reuter) 253

- tomentosus (Reuter) 253

Chlamydatus opacus (Zetterstedt) 19

- wilkinsoni (Douglas \& Scott) 19

Chorosoma schillingi (Schilling) 55

Chroantha ornatula (Herrich-Schäffer) 47

Cinnamus 169

- rhinoceros Distant 169

Codophila varia (Fabricius) 46

Compsidolon 174

Compsonannus 169, 174, 176

- atricornis Linnavuori \& Al-Safadi sp. n. 174-176

- puncticornis Reuter 174

- umbrinus sick! $\rightarrow$ umbrosus 175

- umbrosus Linnavuori \& A1-Safadi sp. n. 174, 175

Coreus marginatus marginatus (Linnaeus) 52

- marginatus syriacus Blöte 52

Coriomeris affinis (Herrich-Schäffer) 53

- vitticollis Reuter 53

Corixa dentipes (Thomson) 19

Corizus fenestella fenestella Horváth 54

- hyoscyami (Linnaeus) 54
Crocistethus waltlianus (Fieber) 37

Cydnus aterrimus (Forster) 38

- hispidulus Klug 38

- sahlbergi Reuter 38

Cyrtopeltis 241

- alkannae Linnavuori 245, 247-250

- asper Linnavuori \& Al-Safadi sp. n. 241, 251

- brunneicollis Linnavuori 247, 249

- callani Odhiambo 249

- echinopis Linnavuori \& Al-Safadi sp. n. 241,245-247, 249

- flavoviridis Linnavuori 248

- kristenseni Poppius 248, 249

- leontion Linnavuori 249

- montivaga Linnavuori 249

- pavoniae Linnavuori 249-251

- volucer (Kirkaldy) 246, 248

- volucer persimilis Poppius 248, 249

- volucer volucer (Kirkaldy) 249

Cyrtopeltis (Campyloneuropsis) asper Linnavuori \& AlSafadi sp. n. 249

Cyrtopeltis (Nesidiocoris) echinopis Linnavuori \& AlSafadi sp. n. 246

Dicranocephalus albipes (Fabricius) 52

- bianchii (Jakovlev) 52

- marginatus (Frey-Gessner) 52

- pallidus (Signoret) 52

- setulosus (Frey-Gessner) 52

Dicyphus constrictus (Boheman) 19

- globulifer (Fallén) 20

Dolycoris baccarum (Linnaeus) 47

Dyroderes umbraculatus (Fabricius) 43

Ellenia 233

Enoplops disciger (Kolenati) 52

Eremocoris plebeius (Fallén) 20

Eurydema fieberi (Schummel) 50

- ornatum (Linnaeus) 49

Eurygaster integriceps Puton 40

Eurystylys 185

- bilqis Linnavuori \& Al-Safadi sp. n. 179, 183-185

- kivuensis Schouteden 185

- marginatus Odhiambo 185

-- pallidiventris Poppius 185

Eysarcoris ventralis (Westwood) 44

Exosehirus sargon Linnavuori 37

- validus (Jakovlev) 37

Geotomus elongatus (Herrich-Schäffer) 38

- intrusus Wagner 39

- pygmaeus (Dallas) 38

Gomphocranum adad Linnavuori 45

- anu Linnavuori 45

- christophi Jakovlev 45

Gonocerus acuteangulatus (Goeze) 52

- juniperi Herrich-Schäffer 52

Graphosoma lineatum italicum (Müller) 41

- semipunctatum (Fabricius) 41

- stali Horváth 41

Holcogaster exilis Horváth 48

Holcostethus congenitus Puchkov 44-46

- vernalis (Wolff) 19, 44, 45

- strictus (Fabricius) 44, 45

Ischnocoris angustulus (Boheman) 20

Leprosoma inconspicuum Bärensprung 40

Liorhyssus hyalinus (Fabricius) 54 
Lutheriella 169

- oecophylloides Poppius 169

Lygocoris rhamnicola (Reuter) 19

Maccavethus corsicus persicus Jakovlev 55

- errans caucasicus (Kolenati) 55

Macrotylus cruciatus (R.F.Sahlberg) 19, 20

Macroscytus brunneus (Fabricius) 38

Marshalliella 233

- obscuricornis Poppius 233

Maurodactylus pictus Wagner 253

Mecidea lindbergi Wagner 41

- pallidissima Jensen-Haarup 41

Megaloceraea recticornis (Geoffroy) 19

Megalocoleus pilosus (Schrank) 19

Melanotrichiella 233

- annulicornis Poppius 233

Micracanthia marginalis (Fallén) 19

Moissonia 233

- kilimana Poppius 234

- punctata (Fieber) 233, 234

- punctata Reuter 233

Mustha spinulosa (Lefebvre) 41

Nabis ericetorum Scholtz 19

- inscriptus (Kirby) 20

- pseudoferus Remane 15

- rugosus (Linnaeus) 20

Nariscus longirostris Linnavuori 54

Neottiglossa bifida (Costa) 44

Nesidiocoris 246

Nezara viridula (Linnaeus) 51

Ochetostethus opacus (Scholtz) 38

Odontotarsus angustatus Jakovlev 39

- armiger Kiritshenko 39

- impictus Jakovlev 39

- purpureolineatus (Rossi) 39

- robustus Jakovlev 39

- rufescens Fieber 39

Odontoscelis 39

- dorsalis (Fabricius) 39

- fuliginosa (Linnaeus) 39

- litura (Fabricius) 39

Omanocoris versicolor (Herrich-Schäffer) 52

Oreocapsus 192

- immundus Linnavuori 192

- vanharteni Linnavuori \& Al-Safadi sp. n. 179, 183, 190, 191

Orthops campestris (Linnaeus) 19

Orthotylus acacicola acacicola Lindberg 186, 187

- acacicola chariensis Linnavuori 186

- acacicola robustus Linnavuori \& Al-Safadi ssp. n. 179, 185,186

- mafraq Linnavuori \& Al-Safadi sp. n. 179, 186, 187

- nassatus (Fabricius) 20

- priesneri-group 186

- virens (Fallén) 20

Palomena mursili Linnavuori 45

Pausias martini (Puton) 51

Peritropis africanus Poppius 181

- armillarius Schmitz 180

- crassicornis Poppius 181

- maculicornis Linnavuori \& Al-Safadi sp. n. 179, 180

Philomyrmex insignis R.F.Sahlberg 20

Phimodera lapponica (Zetterstedt) 20

Phricodus bessaci Villiers 42
Phyllomorpha lacerata Herrich-Schäffer 53

Phytocoris intricatus Flor 20

- kansirob Linnavuori 182

- lineaticollis-group 184

- pini Kirschbaum 20

- populi (Linnaeus) 20

- semicrux Wagner 184

Phytocoris (Eckerleinius) 184

arwa Linnavuori \& Al-Safadi sp. n. 179, 181-184

Phytocoris (Exophytocoris) tithonos Linnavuori \& AlSafadi sp. n. 179-181

Phytocoris (Soosocapsus) 184

Piezodorus lituratus (Fabricius) 51

Pilophorus cinnamopterus (Kirschbaum) 20

Placochilus seladonicus (Fallén) 20

Plagiognathus albipennis (Fallén) 20

- vitellinus (Scholtz) 19

Prionotylus brevicornis (Mulsant \& Rey) 53

Psacasta cypria Puton 40

Psallomimus bicoloripes Wagner 193

- deserticola Wagner 253

- lateralis (Poppius) 193

- tihama Linnavuori \& Al-Safadi sp. n. 179, 191, 192

Psallus ambiguus (Fallén) 20

- graminicola (Zetterstedt) 20

- labeculus Odhiambo 233

Pseumatocoris insignis Kiritshenko 49

Pyrrhocoris apterus (Linnaeus) 55

Rhaphigaster brevispina Horváth 51

- nebulosa (Poda) 50, 51

Rhopalus (Aeschyntelus) maculatus (Fieber) 54

Rhopalus (Rhopalus) subrufus (Gmelin) 54

Riptortus linearis (Fabricius) 54

Salicarus roseri (Herrich-Schäffer) 20

Scantius aegyptius (Linnaeus) 55

- forsteri (Fabricius) 56

Sciocoris capitatus Jakovlev 42

- helferi Fieber 42

- ochraceus Fieber 42

- safavii Hoberlandt 42

- sahlbergi Wagner 43

Sehirus robustus Horváth 37

Seversyia 169

- lupa DeLattre 169

Sigara falleni (Fieber) 20

Spathocera lobata (Herrich-Schäffer) 53

Stagonomus amoenus (Brullé) 45

- bipunctatus (Linnaeus) 45

- devius Seidenstücker 45

Staria lunata (Hahn) 45

Stenotus binotatus (Fabricius) 20

Stictopleurus abutilon pictus (Fieber) 54

- punctatonervosus (Goeze) 20

- ribauti Vidal 55

- riveti Royer 55

Strobilotoma typhaecornis (Fabricius) 53

Strongylocoris niger (Herrich-Schäffer) 20

Syromastus rhombeus (Linnaeus) 52

Tarisa camelus Reuter 40

- subspinosa (Germar) 40

Temnostethus gracilis (Horváth) 20

- pusillus (Herrich-Schäffer) 20

Thaumastella aradoides Horváth 39

Tholagmus Stål 41 
- enkidu Linnavuori 41

Trapezonotus desertus Seidenstücker 19

Trigonotylus fuscitarsis Lammes 19

Tshingisella bella Kiritshenko 40

Tuponia diversa Linnavuori 241, 245, 246

Tuponia (Chlorotuponia) diversa Linnavuori 246

Ventocoris achivus (Horváth) 40

- horvathi Puton 40

- modestus (Jakovlev) 41

- obesus (Stål) 40

Xylocoris formicetorum (Boheman) 20

Zicrona caerulea (Linnaeus) 51

\section{Homoptera}

Acyrthosiphon ignotum Mordvilko 12

Agallia estonica Vilbaste 10

- ribauti Ossiannilsson 10

Amphorophora gei Börner 12

Anoecia zirnitsi Mordvilko 10

Anuraphis subterranea (Walker) 11

Aphis 11

- chloris Koch 11

- idaei van der Groot 33

- lotiradicis Stroyan 11

- pilosellae Börner 11

- tormentillae Passerini 11

Aulacorthrum flavum Börner 33

Brachycaudus 33

Cinara juniperi De Geer 13

Coloradoa absinthii (Lichtenstein) 11

Dysaphis 33

- sorbi Kaltenbach 33

IIyperomyzus lactuae (Linnacus) 32

- rhinanthi (Schouteden) 33

Impatientinum asiaticum Nevsky 11

Mamontova vera Shaposnikov 12

Monaphis antennata (Kaltenbach) 12

Pterocomma 33

Rhopalosiphum padi (Linnaeus) 33

Semiaphis 12

- nolitangere (Aizenberg) 12, 13

Sitobion avenae (Fabricius) 33

Therioaphis (Rhizoberlesia) brachytricha Hille Ris Lambers 12

Therioaphis (Therioaphis) riehmi (Börner) 12

Tinocallis nevskyi Quednau \& Heie 12

\section{Hymenoptera}

Atractodes fennoscandicus remotus Jussila 15

- ruficollis Jussila 13

Chorebus 13

- abnormiceps Nixon 13

- bathyzonus Marshall 13

- gracilis (Nees von Esenbeck) 13

- leptogaster (Haliday) 13

- petiolatus (Nees von Esenbeck) 13

Ephedrus 31-33, 35

- blattnyi Starý 33

- campestris Starý 33

- holmani Starý 33
- minor Stelfox 32

- nitidus Gahan 33

- picticornis Stelfox 32

- plagiator var. minor Stelfox 32

- prociphili Starý 33

- pulchellus Stelfox 33

Ephedrus 31

Ephedrus (Breviephedrus) brevis Stelfox 32, 36

Ephedrus (Ephedrus) cerasicola Starý 32, 35

- chaitophori Gärdenfors 31-33, 35

- helleni Mackauer 32, 36

- koponeni Halme 32, 35

- lacertosus (Haliday) 32, 36

- laevicollis (Thomson) 32, 36

- longistigmus Gärdenfors 31,32, 36

- nacheri Quilis 32, 36

- niger Gautier, Bonnamour \& Gaumont 33, 36

- persicae Froggatt 33, 36

- plagiator (Nees) 33, 36

- vaccinii Gärdenfors 31, 33, 35

Ephedrus (Lysephedrus) validus (Haliday) 32, 34, 36

Formica cunicularia Latreille 13

- rufibarbis Fabricius 13

Lasius flavus (Fabricius) 10

- niger (Linnaeus) 11, 12

Myrmica 12

Pseudopauesia prunicola Halme 33

Toxares 31, 32, 34, 35

- deltiger (Haliday) 34, 36

- flaveolus (Gyrfi) 34

- shigai Takada 34

\section{Lepidoptera}

Eupithecia plumbeolata (Haworth) 14

Neuroptera

Hemerobius micans Olivier 15

Odonata

24 taxa listed on page 167 and discussed on pp. 165-168.

Plecoptera

Amphinemura sulcicollis (Stephens) 217

Diura nanseni (Kempny) 217

Leuctra digitata Kempny 217

\section{Psocoptera}

19 taxa are listed on page 204 and discussed on pp. 201206.

\section{Trichoptera}

Ceratopsyche nevae (Kolenati) 215

Oligostomis reticulata (Linnaeus) 215, 217

Plectrocnemia 217

Rhyacophila nubila (Zetterstedt) 217 